\title{
BMJ Open The NICE-GUT trial protocol: a randomised, placebo controlled trial of oral nitazoxanide for the empiric treatment of acute gastroenteritis among Australian Aboriginal children
}

Claire S Waddington, ${ }^{1}$ Charlie McLeod, ${ }^{2}$ Peter Morris, ${ }^{3,4}$ Asha Bowen, ${ }^{2,3}$ Mark Naunton, ${ }^{5}$ Jonathan Carapetis,,${ }^{1,2}$ Keith Grimwood, ${ }^{6}$ Roy Robins-Browne, ${ }^{7}$ Carl D Kirkwood, ${ }^{8}$ Robert Baird, ${ }^{9}$ David Green, ${ }^{10}$ Ross Andrews, ${ }^{3}$ Deborah Fearon, ${ }^{10}$ Joshua Francis, ${ }^{3,4}$ Julie A Marsh, ${ }^{1,11}$ Thomas Snelling ${ }^{1}$

To cite: Waddington CS, McLeod C, Morris P, et al. The NICE-GUT trial protocol: a randomised, placebo controlled trial of oral nitazoxanide for the empiric treatment of acute gastroenteritis among Australian Aboriginal children. BMJ Open 2018;8:e019632. doi:10.1136/ bmjopen-2017-019632

- Prepublication history and additional material for this paper are available online. To view these files, please visit the journal online (http://dx.doi. org/10.1136/bmjopen-2017019632).

Received 14 September 2017 Revised 27 November 2017 Accepted 7 December 2017

Check for updates

For numbered affiliations see end of article.

Correspondence to Dr Thomas Snelling: tom.snelling@telethonkids. org.au

\section{ABSTRACT}

Introduction Diarrhoeal disease is the second leading cause of death in children under 5 years globally, killing 525000 annually. Australian Aboriginal and Torres Strait Islander (hereafter Aboriginal) children suffer a high burden of disease. Randomised trials in other populations suggest nitazoxanide accelerates recovery for children with Giardia, amoebiasis, Cryptosporidium, Rotavirus and Norovirus gastroenteritis, as well as in cases where no enteropathogens are found.

Methods and analysis This double blind, 1:1 randomised, placebo controlled trial is investigating the impact of oral nitazoxanide on acute gastroenteritis in hospitalised Australian Aboriginal children aged 3 months to $<5$ years. Dosing is based on age-based dosing. The primary endpoint is the time to resolution of 'significant illness' defined as the time from randomisation to the time of clinical assessment as medically ready for discharge, or to the time of actual discharge from hospital, whichever occurs first. Secondary endpoints include duration of hospitalisation, symptom severity during the period of significant illness and following treatment, duration of rehydration and drug safety. Patients will be followed for medically significant events for 60 days. Analysis is based on Bayesian inference. Subgroup analysis will occur by pathogen type (bacteria, virus or parasite), rotavirus vaccination status, age and illness severity.

Ethics and dissemination Ethics approval has been granted by the Central Australian Human Research Ethics Committee (HREC-14-221) and the Human Research Ethics Committee of the Northern Territory Department of Health and Menzies School of Health Research (HREC2014-2172). Study investigators will ensure that the trial is conducted in accordance with the principles of the Declaration of Helsinki. Individual participant consent will be obtained. Results will be disseminated via peer-reviewed publication. Trial registration number ACTRN12614000381684.

\section{Strengths and limitations of this study}

- NICE-GUT is the first study in Australia to evaluate the impact of nitazoxanide on acute gastroenteritis in Aboriginal children.

- This pragmatic randomised control trial is based on Bayesian adaptive design, an innovative trial methodology that overcomes some of the limitations encountered in trials informed by frequentist design.

- While Bayesian adaptive trials are becoming increasingly popular, they are yet to be established and accepted as routine research practice.

- The investigator group will rapidly ensure translation of research findings into clinical practice and health policy.

Loss to follow-up is likely to be encountered in this study.

\section{INTRODUCTION}

Acute gastroenteritis is the leading cause of global childhood mortality and morbidity, and disproportionately affects those in resource-poor settings. ${ }^{1}$ The GEMS study ${ }^{2}$ quantified the pathogen-specific burden of enteric disease in several high burden low-income countries; in that study, infection with enterotoxigenic and enteropathogenic Escherichia coli in infants 0-11 months and Cryptosporidium in toddlers 12-23 months was associated with an increased 60-day mortality. Australian Aboriginal children also experience a disproportionate burden of disease compared with non-Aboriginal children. ${ }^{3}$ Although the introduction of rotavirus vaccination in 2006 has reduced disease burden, vaccine effectiveness in Aboriginal children has been low compared with that observed in non-Aboriginal Australian children. ${ }^{4}$ 
Gastroenteritis outbreaks are well described among Aboriginal children living in remote central and northern Australia. These epidemics place enormous strain on remote health clinics, aeromedical retrieval services and hospital facilities. ${ }^{5}{ }^{6}$ An effective oral treatment for the management of gastroenteritis would have a substantial impact in this setting in reducing disease burden and potentially reducing sequelae associated with prolonged and recurrent intestinal infections such as malnutrition, stunting and cognitive impairment. ${ }^{78}$ Therefore, while disease prevention remains the long-term goal, effective treatment strategies are still needed to reduce the health, social and economic and impact of childhood gastroenteritis.

Nitazoxanide, a thiazolide antimicrobial developed in the 1980s, has been shown to have broad-spectrum in vitro and/or clinical activity against a wide range of enteric pathogens including Giardia, Entamoeba, Bacteroides, Clostridium, Cryptosporidium, Rotavirus and Norovirus. $^{9-15}$ Nitazoxanide is administered orally, is heat stable, and has proven to be safe and well tolerated in children, ${ }^{9}$ making it suitable for use in remote and low resource settings. To date, clinical trials of nitazoxanide have been conducted in low and middle income countries, where data have emerged to support its empirical use for the treatment of acute gastroenteritis regardless of aetiology. ${ }^{9}$ Results of these trials cannot be automatically extrapolated to our high-income (but high disease burden) setting.

Here we describe a clinical trial protocol to investigate if nitazoxanide is clinically beneficial for the empirical treatment of acute gastroenteritis among Australian Aboriginal children. Clinical trials in high burden and often low resourced settings are challenging. It is important to strike a balance between gathering reliable data pertaining to relevant endpoints against the challenges of working with a disparate and culturally diverse population for whom English language literacy and understanding of research may be limited and in whom pragmatic public health solutions are needed in a timely manner. By using the principals of pragmatic trial design, cross-cultural working and a Bayesian adaptive statistical approach, this trial will provide timely, relevant data to inform the management of gastroenteritis in these children.

\section{METHODS AND ANALYSIS \\ Design}

NICE-GUT is a double-blind, randomised, placebo-controlled efficacy trial of oral nitazoxanide in Aboriginal children hospitalised with acute gastroenteritis. The protocol for enrolment, intervention, end points and analysis are based on the principles of pragmatic trial design and Bayesian sample-size adaptation; see trial flow chart (figure 1) and trial events schedule (table 1).

\section{Study setting}

The study is being conducted at The Royal Darwin Hospital and Alice Springs Hospital, the two principal hospitals serving the Northern Territory (NT) of Australia. Aboriginal people represent $30 \%$ of the population in the NT. ${ }^{16}$ Most of the NT is classified as extremely remote; hospitalisation frequently requires aeromedical retrieval. Diarrhoea is the most prevalent condition at admission among infants and young adults in the NT, occurring in around 300 admissions per 1000 Aboriginal population. ${ }^{3}$ Epidemics of gastroenteritis are also well described in this population. $^{5}$

\section{Objectives}

The primary objective of the NICE-GUT trial is to determine whether empiric treatment with oral nitazoxanide (NTZ), compared with placebo, reduces the duration of significant illness in Aboriginal children hospitalised for acute gastroenteritis.

\section{Outcomes}

The primary outcome is 'the time period of significant illness', defined as the time from randomisation until either (1) the time to achieve a score $\geq 2$ on the "medical readiness for discharge' scoring system (table 2) as assessed by the study doctor or study nurse or (2) the time to actual hospital discharge, except for children who die, abscond or are transferred to other health facilities, whichever is sooner. Assessment of medical readiness for discharge is based on medical factors only and specifically excludes social issues that may influence actual discharge decisions. Further details are provided under study procedures.

Secondary outcomes include (1) duration of hospitalisation, defined as the time from randomisation until actual discharge from hospital (including time after being assessed as medically suitable for discharge); (2) number of stools and vomiting episodes during the 'time period of significant illness'; (3) the presence and severity of symptoms from the time of randomisation to day 7 , based on the number of vomits/diarrhoea, overall symptoms and activity level; (4) the presence and severity of dehydration based on WHO guidelines (online supplementary appendix 1$)^{17}$; and (5) the time between starting intravenous, intraosseous or nasogastric rehydration or randomisation (whichever occurs later) and ceasing rehydration.

Safety outcomes include adverse events attributed to the study drug, mortality at 60 days, recurrent gastroenteritis within 60 days of enrolment, new onset malnutrition (not pre-existing) requiring medical assessment/intervention within 60 days of enrolment and prolongation of symptoms beyond 7 days.

\section{Participants}

Potential participants are identified from review of ward admission logs, hospital information systems and by hospital clinical staff. A verbal overview of the study, aided by use of a study 'flip-chart' giving a pictorial outline of the study, is used to outline the study to the parent/ legallyresponsible carer of potential participants. A written information sheet is also provided. This approach 


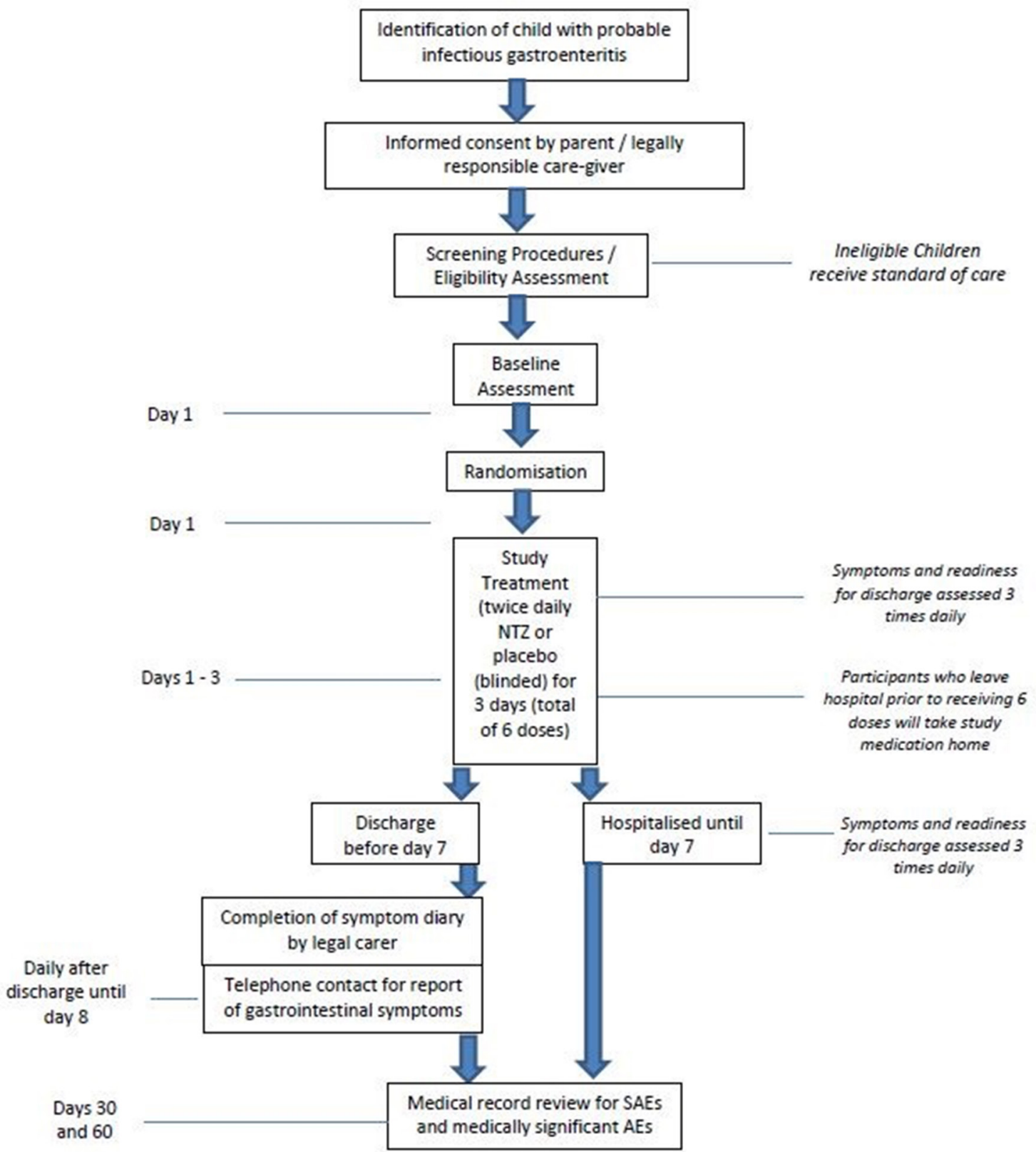

Figure 1 Trial flow chart. SAE, serious adverse events.

was informed by consultation with Aboriginal people. Administration of the study drug must occur within 12 hours of all screening procedures.

Australian Aboriginal children aged $\geq 3$ months and $<5$ years hospitalised for less than 48 hours with a primary diagnosis of acute gastroenteritis (in the opinion of the admitting doctor or the study nurse) are eligible for enrolment. Data exist regarding the safety of nitazoxanide in this age group, ${ }^{9} 111318-21$ but not in infants $<3$ months old. Ethnicity is determined by self-report of the parent or legally responsible carer. Additional requirements for eligibility include permission by the parent to (1) notify healthcare practitioners involved in their child's care about participation, (2) ascertain vaccination history from the Australian Immunisation Register and/or local provider, and (3) obtain medical data from the medical records and/or primary care provider. Exclusion criteria are (1) bloody diarrhoea (dysentery) or known infection with an enteric pathogen requiring alternative antimicrobial treatment, (2) clinical suspicion of a non-infectious aetiology or an intestinal obstruction, (3) contraindication or allergy to nitazoxanide, (4) previous enrolment, (5) symptom duration >14 days, (6) non-availability of oral route for drug administration.

\section{Study procedures}

Baseline assessment

To assess eligibility and to allow description of the study population, demographic data (including number of co-inhabitants $<5$ years and $\geq 5$ years old and number of bedrooms in the household), clinical details of current illness, past medical history (including rotavirus 


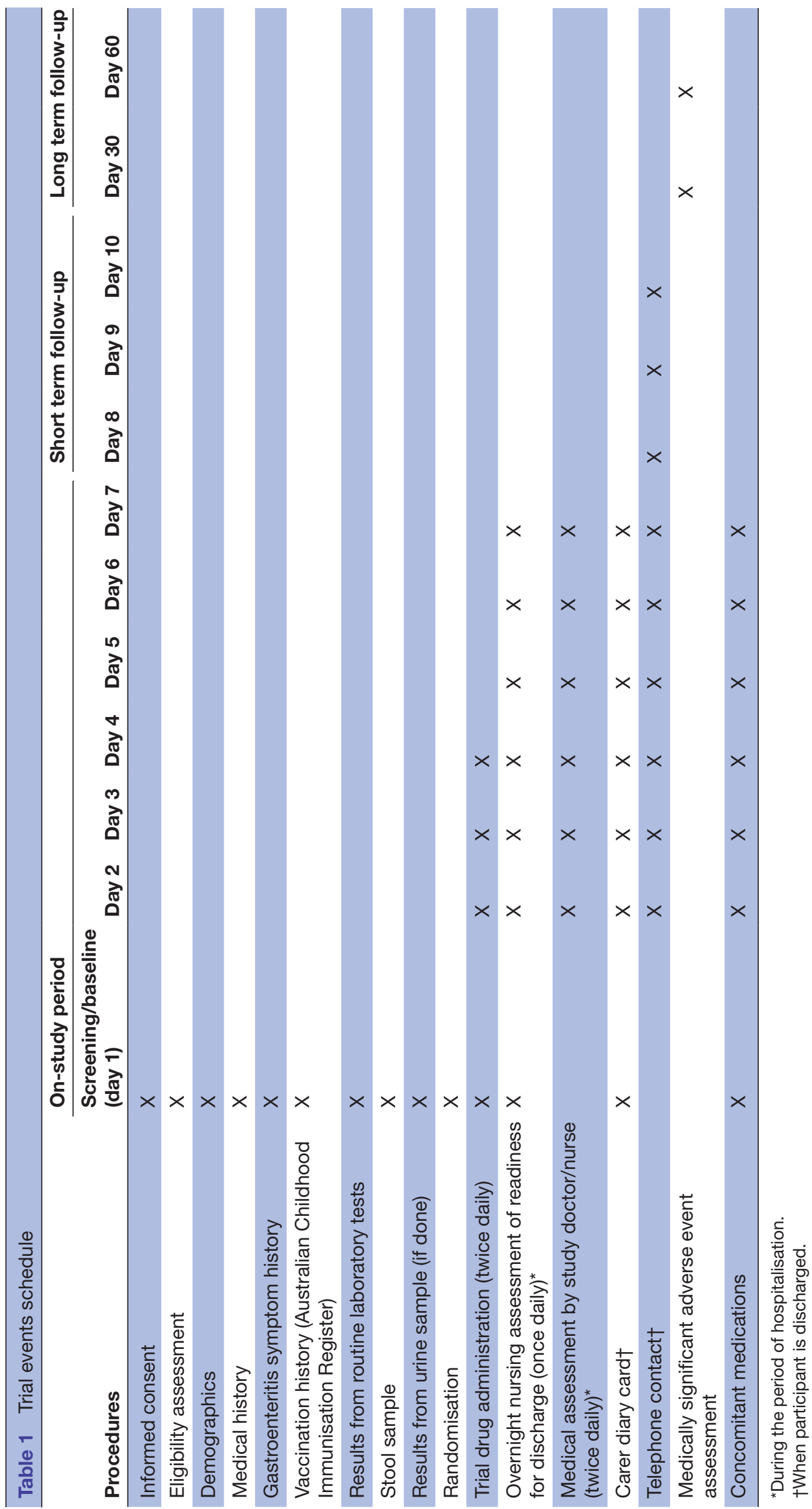

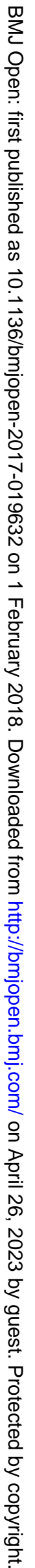


Table 2 Medical readiness for discharge scoring system

\begin{tabular}{|c|c|c|c|}
\hline \multicolumn{4}{|l|}{ Score } \\
\hline 0 & 1 & 2 & 3 \\
\hline $\begin{array}{l}\text { Not medically } \\
\text { ready for } \\
\text { discharge. } \\
\text { Ongoing } \\
\text { requirement } \\
\text { for hospital } \\
\text { level care }\end{array}$ & $\begin{array}{l}\text { Not medically } \\
\text { ready for } \\
\text { discharge, } \\
\text { but improved } \\
\text { from } \\
\text { condition at } \\
\text { randomisation }\end{array}$ & $\begin{array}{l}\text { Medically ready } \\
\text { for discharge } \\
\text { with suitable } \\
\text { carer input, } \\
\text { but not fully } \\
\text { recovered }\end{array}$ & $\begin{array}{l}\text { Medically } \\
\text { ready for } \\
\text { discharge, } \\
\text { fully } \\
\text { recovered }\end{array}$ \\
\hline
\end{tabular}

vaccination history), baseline bowel pattern, breastfeeding status and anthropometric indices (bare weight, length/ height mid-upper arm circumference) are gathered.

Randomisation, intervention and blinding

Randomisation of eligible participants is by computer generated allocation sequence, using randomly permuted block sizes, with stratification by age $(<1$ year vs $\geq 1$ year old), hospital and remoteness of residence (urban Darwin or Alice Springs vs elsewhere in the NT) on the night prior to admission.

Participants are randomised 1:1 within the four strata to receive either nitazoxanide or placebo. The study drug is prescribed to be given within 12 hours of randomisation, with nitazoxanide dose determined by age-based dosing ( $<1$ year olds: $7.5 \mathrm{mg} / \mathrm{kg} /$ dose; 1 to 3 years old: $100 \mathrm{mg} /$ dose; $\geq 4$ years of age, $200 \mathrm{mg} /$ dose) and administered twice daily for 3 days orally or by nasogastric/ enteric tube. If vomiting occurs within $15 \mathrm{~min}$ of administration, the drug is re-administered. If the child vomits a second time, no further doses will be given. The placebo is physically and chemically identical to the intervention drug except it does not contain nitazoxanide. All investigators, research staff and participants, except for the trial statistician, are blinded to the treatment allocation until study completion. Nitazoxanide is the standard treatment for refractory cryptosporidiosis in our setting. There is provision for unblinded nitazoxanide rescue therapy for participants with refractory cryptosporidiosis after discussion with the co-ordinating principal investigator. Transfer to rescue treatment does not require withdrawal of the participant from the study.

All aspects of patient management such as rehydration, management of ongoing losses and provision of oral/ enteral/parenteral diet and management of comorbidites (eg, hypokalaemia/hyperkalaemia or hypoglycaemia) are managed as per the instruction of the primary treating team in accordance with local guidelines.

\section{Follow-up}

Participants are reviewed by a study nurse twice daily (08:00+4 hours and 16:00+4 hours) during the first 7 days of hospitalisation to record symptoms (including number of diarrhoeal stools and vomiting episodes), activity level, hydration status, any adverse events and whether the child is medically ready for discharge (see table 2 ).
Volume and route of re-hydration therapy and use of unblinded 'rescue' nitazoxanide are recorded once daily. A further assessment of readiness for discharge is requested of the ward nurses daily $(0000+4$ hours); if a child is judged medically ready for discharge by a ward nurse overnight, this is validated by a study nurse or study doctor the following morning.

Participants discharged from hospital prior to study day 7 are asked to complete an aide memoire diary card detailing administration of study drug and symptoms. Participants for whom phone contact cannot be made by day 10 are considered lost to follow-up, but remain enrolled. Participants who remain hospitalised at day 7 are followed daily until discharge to determine the reason for ongoing hospitalisation. Any new illness prolonging hospitalisation is reported as a serious adverse event (SAE).

At study day 30 and day 60, electronic hospital and primary care records are reviewed by a study nurse to ascertain the safety endpoints. The trial will end following completion of the last laboratory assay on the last participant enrolled, or 60 days postrandomisation of the last participant, whichever is later.

\section{Data collection}

Relevant data are obtained from hospital and laboratory records, participant diaries, radiographs and clinical correspondence. Data are recorded onto paper case report forms. Participants are followed up at day 7 after enrolment by a study nurse (by telephone if discharged) to ascertain symptoms. Once collected, data are entered onto a password protected electronic data capture system built in Medrio (mEDU Academic eClinical, 2017). Study documents will be stored securely at each study site and will be archived when the youngest participant reaches 25 years of age.

\section{Laboratory testing}

A stool sample $(>3 \mathrm{~mL})$ is obtained as soon as possible after randomisation. Stools undergo wet preparation evaluation and routine bacterial culture on MacConkey and Xylose Lysine Deoxycholate agar as well as Campylobacter-specific media. A blind sweep of the MacConkey agar plate is collected after 24 hours of incubation and inoculated onto dorset egg medium, incubated overnight at $37^{\circ} \mathrm{C}$, and then at room temperature until transfer to the University of Melbourne for molecular testing for enterovirulent pathotypes of E. coli. Cryptosporidium and Giardia are identified by rapid faecal antigen testing. Stool remaining after routine laboratory testing is frozen at $<-70^{\circ} \mathrm{C}$ before being transferred to the Murdoch Children's Research Institute for testing for rotavirus and norovirus by PCR.

Other clinically indicated laboratory tests including blood culture, biochemistry and haematology are performed at the discretion of the treating team and according to standard hospital procedures. 


\section{Withdrawal of participants}

Parents and legal carers have the right to withdraw their child from the trial at any time. The chief investigator (CI) or site principal investigator may elect to discontinue participant involvement in the trial if (1) the child is found to be ineligible following recruitment, (2) significant protocol violations occur, (3) non-adherence with the treatment regimen or trial requirements occur, (4) adverse events arise requiring discontinuation of the trial medication, (5) withdrawal of consent occurs or (6) loss to follow-up occurs. Data obtained prior to participant withdrawal from the study will be included in the analysis.

\section{Data analyses and sample size justification}

Participants are analysed according to the group they are randomised to (intention-to-treat analysis). All randomised participants with observed outcome data will be analysed in a secondary completers' population analysis. Analyses are based on Bayesian inference; the statistical model considers the evidence accumulated during the trial and the assumed prior knowledge (known as a prior distribution). The parameters in the model are assigned non-informative prior distributions. This approach was used to ensure the trial continued only as long as there remained reasonable uncertainty regarding the treatment effect, thereby aiding timely trial results, potentially accelerating implementation and minimising exposure to any risk and research burden. This method of statistical analysis differs from traditional frequentist trials in which inferences are based on the likelihood of different sample outcomes against a null hypothesis. In contrast, Bayesian analysis is done conditional on the observed data, but inference does not depend on either the sampling design or on outcomes that did not occur.

The variables for which adjustment are made are age group and remoteness. Outcomes for these strata are reported separately, but the model captures any commonalities across strata. The statistical model allows evidence relating to the effectiveness of an intervention in one stratum to contribute (via 'borrowing') to the estimation of the posterior probability in the other strata, but this only occurs to the extent that the intervention effect is similar across different strata. The results of these analyses are used to achieve the primary objective of the trial which is to determine the effectiveness of nitazoxanide and the extent to which that effectiveness varies between strata (intervention-stratum interaction).

A first interim analysis occurs after the 126th randomised child has recorded information for time of significant medical illness. If required, further interim analyses will occur as soon as practicable after every additional 20 children enrolled (the 150th, 170th, 190th, 210th, 230th, 250th, 270th and 300th enrolment). At each interim analysis, the Bayesian predictive probability that the time of significant medical illness is shorter with nitazoxanide treatment versus placebo is estimated for each stratum. The estimated probability for each stratum determines whether to
1. stop enrolment for futility $(<20 \%$ or $<40 \%$ probability for interim analyses $1-4$ and 5-8, respectively, that nitazoxanide shortens the duration of significant illness compared with placebo;

2. stop enrolment for success $(>99 \%$ or $>98 \%$ probability for interim analyses $1-4$ and 5-8, respectively, that nitazoxanide shortens the duration of significant illness compared with placebo;

3. continue enrolment due to uncertainty of effect (20\%-99\% or between $40 \%$ and $98 \%$ probability for interim analyses $1-4$ and 5-8, respectively, that nitazoxanide shortens the duration of significant illness compared with placebo.

If none of the stopping rules have been met after 300 children have been enrolled, no further children will be enrolled and the nitazoxanide arm will be considered superior for the primary endpoint if there is $>97.5 \%$ probability that this arm has a shorter time of significant medical illness compared with the placebo arm.

The comparison of the nitazoxanide and placebo arms for time to resolution of significant medical illness (primary outcome) is summarised by a hazard ratio (HR) and denoted by $\theta \mathrm{d}$ (or $\log$ transformed $\theta \mathrm{d}$ for the $\log$ HR). The hypothesis test for the comparison between nitazoxanide and placebo arms is

$$
\begin{gathered}
H_{0}: \theta_{d} \leq 1\left(\text { alternatively } \log \left(\theta_{d}\right) \leq 0\right) \\
H_{1}: \theta_{d}>1\left(\text { alternatively } \log \left(\theta_{d}\right)>0\right)
\end{gathered}
$$

The log HR (treatment effect) is given a vague non-informative prior, $\log \left(\theta_{0 \mathrm{~d}}\right) \sim \mathrm{N}[0,10]$ : in particular, the prior probability that nitazoxanide is beneficial is the same as the prior probability that it is futile. If there is a high posterior probability that the treatment effect, $\log \theta \mathrm{d}$, is positive, the treatment is declared to be efficacious. The posterior probability is conditional on the final results from all subjects enrolled in the trial.

The stopping rules for the primary analysis have been calibrated to ensure a one-sided experiment-wise type 1 error rate of 0.05 . All prespecified analyses will be reported in the main manuscript.

Subgroup analysis will occur according to pathogen group (bacteria, virus or parasite), rotavirus vaccination status, age $(<1$ and $\geq 1$ year) and severity of illness at presentation (nil, mild, moderate or severe acidosis or dehydration) (online supplementary appendix 1 and 2).

\section{Safety reporting and trial oversight}

All SAEs are recorded from the time of enrolment until the 60-day follow-up, and are reported to the sponsor within one working day. All SAEs are reported to the approving ethics committees in accordance with their requirements. All suspected, unexpected serious adverse reactions are reported to the Australian Therapeutic Goods Administration by the sponsor within 7 days.

A Data and Safety Monitoring Committee convenes at least twice per year to provide safety oversight and to monitor the overall conduct of the trial including adherence to stopping rules prespecified in the protocol. 
Randomisation codes are held by the trial statistician and password protected. Code breaks are only to be used if a situation arises where the CI deems it necessary to break the blinding process for compelling medical or safety reasons.

\section{DISCUSSION}

Despite the advent of rotavirus vaccination, gastroenteritis remains a significant health problem in Australian Aboriginal children, as well as in many resource-poor settings globally. Treatment that reduces the duration and severity of disease is needed to reduce the health, economical and societal burden of disease.

Clinical trials in remote populations and high disease burden settings present many logistical challenges. This protocol outlines our approach to meeting several of these challenges. Pragmatic clinical trials recognise that although randomisation, blinding and precise endpoints promote internal validity, they do not ensure external validity-that is, extrapolation of trial outcomes to populations and situations beyond the trial population. For trials such as this that seek to address important global health issues, it is essential that data are gathered in a way that facilitates extrapolation beyond the confines of the specific protocol and trial population.

In this trial, we are gathering a range of baseline data to allow full description of the study population and, where numbers permit, subgroup analyses that may inform the wider global health agenda. We selected a primary endpoint based on duration of significant illness (requiring hospitalisation) as the most important endpoint because of its relevance to patients, families, care providers and policy makers; we believe that it will be informative for other settings.

Other trials of nitazoxanide have restricted treatment to those with confirmed infection with one or more specific pathogens. For nitazoxanide to be broadly applicable for treatment of Aboriginal children, it will need to be used empirically, that is, before stool microbiology results are available. This is because awaiting stool results will delay commencement of therapy; because of these delays, children with acute gastroenteritis treated remotely usually have no microbiology testing. Our eligibility criteria are very inclusive; we aim to include all children with presumed infective gastroenteritis except those children ineligible because of known or anticipated intolerance or where alternative therapy is indicated. We have prespecified subgroups analyses based on age and remoteness because the spectrum of pathogens is somewhat different across these patient groups; detection of group-specific treatment effects could inform targeted treatment based on these factors.

Ensuring valid informed consent that meets the standards outlined in the Declaration of Helsinki is of paramount importance, especially in a vulnerable population in whom much historical research has resulted in little direct benefit. It can be difficult to ensure this in the context of low levels of health literacy and when working across cultures where concepts of individual versus community-level determination differ. We sought involvement of an Aboriginal reference group to inform the study design and consent procedures.

Traditional 'frequentist' methods for analysing clinical trials demand that the sample size be determined in advance, based on the expected treatment response rate among controls and a largely arbitrary decision about what constitutes a minimum clinically important difference. Such judgements are based on a 'best guess' approach, and consequently most trials either fail to answer the question they set out to address with certainty or, alternatively, continue to enrol participants far beyond the point where evidence of an effect has been reached. Adaptation of the sample size based on accumulated data and Bayesian inference, as used in this trial, will increase the probability of a conclusive result while ensuring that the trial does not continue beyond the point where either futility or success can be concluded. This will help minimise any risk and burden associated with research participation, ensure that the trial findings, if positive, are translated into practice in a timely fashion and expedite evaluation of alternative therapies if the trial results are negative.

\section{ETHICS AND DISSEMINATION}

Study investigators will ensure that this trial is conducted in accordance with the principles of the Declaration of Helsinki ${ }^{22}$ and with the ICH Guidelines for Good Clinical Practice. $^{23}$

Dissemination of results will occur through peer-reviewed publications and presentation(s) to important stakeholders.

\section{Earlier protocol versions}

After 126 enrolments, before any analysis and while all investigators including the trial statistician remained blinded, a substantial amendment to the protocol was made due to slower than anticipated enrolment. In the earlier version of the trial protocol, the study procedures and endpoints were the same, except we planned for a single frequentist time-to-event analysis after a fixed sample size of 400 enrolments.
Author affiliations
${ }^{1}$ Wesfarmers Centre of Vaccines and Infectious Diseases, Telethon Kids Institute, Subiaco, Australia
${ }^{2}$ Infectious Diseases Department, Princess Margaret Hospital for Children, Perth, Australia
${ }^{3}$ Menzies School of Health Research, Casuarina, Australia
${ }^{4}$ Department of Paediatrics, Royal Darwin Hospital, Darwin, Australia
${ }^{5}$ Department of Pharmacy, University of Canberra, Bruce, Canberra, Australia
${ }^{6}$ Queensland Children's Medical Research Institute, Brisbane, Queensland, Australia ${ }^{7}$ Department of Microbiology and Immunology, University of Melbourne, Melbourne, Victoria, Australia
${ }^{8}$ Murdoch Children's Research Institute, Parkville, Victoria, Australia
${ }^{9}$ Department of Microbiology, Royal Darwin Hospital, Darwin, Australia
${ }^{10}$ Department of Paediatrics, Alice Springs Hospital, Alice Springs, Australia 
${ }^{11}$ Centre for Applied Statistics, University of Western Australia, Crawley, Australia

Acknowledgements The authors acknowledge the support of Romark Laboratories for providing the study drug and placebo for unrestricted use. The authors also acknowledge the contribution of the independent data and safety monitoring committee, Professor Don Robertson, Professor John Crump, A/ Professor Andrew Steere, A/Professor Timothy Spelman and A/Professor Rob Roseby; the willing study participants and their families; the Aboriginal Child Health Reference Group of the Menzies School of Health Research; the staff of the Royal Darwin Hospital and Alice Springs Hospitals, particularly Dr Deborah Fearon, Dr Joshua Francis, Dr Keshan Satharasinghe, and Mr James McLeod, Ms Sharon Trosonco, Mrs Carly McCallum and Ms Jane Nelson.

Contributors CSW and CM contributed equally to preparation of the manuscript. CSW developed the study processes and procedures and primarily authored the initial trial protocol. TS conceived the study and, with JAM, authored the amendment to a Bayesian approach. $\mathrm{CDK}, \mathrm{RR}-\mathrm{B}$ and $\mathrm{RB}$ were primarily responsible for the microbiological analysis procedures. PM, AB, MN, JC, DG, DF, JF and KG contributed to the clinical aspects of the protocol. RA and KG contributed to the design. All authors have reviewed and approved the manuscript. All authors meet the BMJ uniform requirements for authorship inclusion and have seen and approved the final version of this manuscript.

Funding This work was supported by The National Health Medical Research Council (1069772). Fellowship support was provided by The National Health Medical Research Council (111657 and 1088735) and the Channel 7 Telethon Foundation.

Competing interests None declared.

Patient consent Not required.

Ethics approval Ethics approval has been granted by the Central Australian HREC (HREC-14-221) and the HREC of the NT Department of Health and Menzies School of Health Research (HREC-2014- 2172). Planned deviations from this protocol will not occur without approval from the relevant governing bodies.

Provenance and peer review Not commissioned; externally peer reviewed.

Open Access This is an Open Access article distributed in accordance with the Creative Commons Attribution Non Commercial (CC BY-NC 4.0) license, which permits others to distribute, remix, adapt, build upon this work non-commercially, and license their derivative works on different terms, provided the original work is properly cited and the use is non-commercial. See: http://creativecommons.org/ licenses/by-nc/4.0/

(c) Article author(s) (or their employer(s) unless otherwise stated in the text of the article) 2018. All rights reserved. No commercial use is permitted unless otherwise expressly granted.

\section{REFERENCES}

1. World Health Organisation and UNICEF. The Integrated Global action plan for Pneumonia and Diarrhoea (GAPPD). 2013. http://apps. who. int/iris/bitstream/10665/79200/1/9789241505239_eng.pdf?ua=1 (accessed 13 Aug 2017).

2. Kotloff KL, Nataro JP, Blackwelder WC, et al. Burden and aetiology of diarrhoeal disease in infants and young children in developing countries (the Global Enteric Multicenter Study, GEMS): a prospective, case-control study. Lancet 2013;382:209-22.

3. Li SQ, Guthridge S, Tursan d'Espaignet E, et al. From infancy to young adulthood: health status in the Northern Territory. Darwin: Department of Health and Community Services, 2006. http:// digitallibrary.health.nt.gov.au/dspace/bitstream/10137/84/1/infancy to_young_adulthood_2006.pdf (accessed 13 Aug 2017).
4. Dey A, Wang H, Menzies R, et al. Changes in hospitalisations for acute gastroenteritis in Australia after the national rotavirus vaccination program. Med J Aust 2012;197:453-7.

5. Armstrong P. Rotaviral gastroenteritis in the NT: a description of the epidemiology 1995-2001 and future directions for research. Northern Territory Disease Control Bulletin 2001;8:1-5.

6. Gelbart B, Hansen-Knarhoi M, Binns P, et al. Rotavirus outbreak in a remote Aboriginal community: the burden of disease. $J$ Paediatr Child Health 2006;42:775-80.

7. Niehaus MD, Moore SR, Patrick PD, et al. Early childhood diarrhea is associated with diminished cognitive function 4 to 7 years later in children in a northeast Brazilian shantytown. Am J Trop Med Hyg 2002;66:590-3.

8. Guerrant DI, Moore SR, Lima AA, et al. Association of early childhood diarrhea and cryptosporidiosis with impaired physical fitness and cognitive function four-seven years later in a poor urban community in northeast Brazil. Am J Trop Med Hyg 1999;61:707-13.

9. Rossignol J-F, Lopez-Chegne N, Julcamoro LM, et al. Nitazoxanide for the empiric treatment of pediatric infectious diarrhea. Trans $R$ Soc Trop Med Hyg 2012;106:167-73.

10. Rossignol JF. Nitazoxanide in the treatment of acquired immune deficiency syndrome-related cryptosporidiosis: results of the United States compassionate use program in 365 patients. Aliment Pharmacol Ther 2006;24:887-94.

11. Rossignol JF, Abu-Zekry M, Hussein A, et al. Effect of nitazoxanide for treatment of severe rotavirus diarrhoea: randomised double-blind placebo-controlled trial. Lancet 2006;368:124-9.

12. Rossignol J-F, Kabil SM, El-Gohary Y, et al. Effect of nitazoxanide in diarrhea and enteritis caused by cryptosporidium species. Clin Gastroenterol Hapatol 2006;4:320-4.

13. Rossignol JF, Kabil SM, El-Gohary Y, et al. Nitazoxanide in the treatment of amoebiasis. Trans $R$ Soc Trop Med Hyg 2007;101:1025-31.

14. Rossignol JF, Lopez-Chegne N, Julcamoro LM, et al. Nitazoxanide for the empiric treatment of pediatric infectious diarrhea. Trans $R$ Soc Trop Med Hyg 2012;106:167-73.

15. Rossignol JF, El-Gohary YM. Nitazoxanide in the treatment of viral gastroenteritis: a randomized double-blind placebo-controlled clinical trial. Aliment Pharmacol Ther 2006;24:1423-30.

16. Department of Health. Northern Territory resident population estimates by age, sex, indigenous status and health districts (1971-2016)', prepared by health gains planning, file updated on 27 February 2017: ABS Estimated Resident Population, 2017.

17. World Health Organization. The treatment of diarrhoea. 2005 http:// whqlibdoc.who.int/publications/2005/9241593180.pdf

18. McLeod C, Morris PS, Snelling TL, et al. Nitazoxanide for the treatment of infectious diarrhoea in the Northern Territory, Australia 2007-2012. Rural Remote Health 2014;14:2759.

19. Teran CG, Teran-Escalera CN, Villarroel P. Nitazoxanide vs. probiotics for the treatment of acute rotavirus diarrhea in children: a randomized, single-blind, controlled trial in Bolivian children. Int Infect Dis 2009;13:518-23.

20. Escobedo AA, Alvarez G, González ME, et al. The treatment of giardiasis in children: single-dose tinidazole compared with 3 days of nitazoxanide. Ann Trop Med Parasitol 2008;102:199-207.

21. Juan JO, Lopez Chegne N, Gargala G, et al. Comparative clinical studies of nitazoxanide, albendazole and praziquantel in the treatment of ascariasis, trichuriasis and hymenolepiasis in children from Peru. Trans R Soc Trop Med Hyg 2002;96:193-6.

22. World Medical Association. World medical association declaration of Helsinki: ethical principles for medical research involving human subjects. JAMA 2013;310:219104.

23. International Council for Harmonisation. Guidelines for good clinical practice, 2016. http://www.ich.org/fileadmin/Public_Web_Site/ICH Products/Guidelines/Efficacy/E6/E6_R2_Step_4.pdf (accessed 4 Sep 2017). 